\title{
Multiple Constrained Dynamic Path Optimization based on Improved Ant Colony Algorithm
}

\author{
Seng Dewen*, Tang Meixia, Wu Hao, Fang Xujian and Xu Haitao \\ School of Software Engineering, Hangzhou Dianzi University, 310018, Hangzhou, \\ China
}

\begin{abstract}
Vehicle navigation system can effectively alleviate traffic congestion, reduce pollution, and reduce travel cost and other problems. As is known to all, the traditional ones are all just static path planning with problems of not only weak effectiveness but also lack of standard optimal path options. They usually provide only one path which represents the shortest time or shortest distance, and ignore the actual demands of the dirivers. Based on traffic data of the past, the upcoming traffic flows can be estimated. With the help of the improved ant colony algorithm, the dynamic optimal path planning results will meet the need of the travelers according with multiple actual constraints.
\end{abstract}

Keywords: Intelligent transportation; vehicle navigation system; improved ant colony algorithm; optimal multiple constrained dynamic path

\section{Introduction}

Traffic congestion has not only become the biggest problem of urban residents travelling, but also become the biggest bottlenecks in urban development [1-3]. With the rapid development of modern cities, traditional ways like road infrastructure, traffic restrictions based on the last digit of license plate numbers and traffic control have been struggling to resolve traffic congestion for long. Therefore, a more orderly and efficient solution must appear, and the development of intelligent transportation had proposed a new idea for the issue. Intelligent navigation based on the dynamic path planning in solving the problem is a very good application [4-9]. As the rapid development of computer technologies and

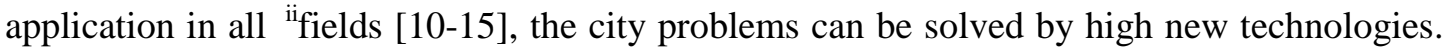
The intelligent navigation base on dynamic path planning in solving this problem has a very good application, mainly combine the urban road network topological relationship, historical traffic data and real-time traffic information to planning a dynamic path strategy which best meet travelers' mind. And thus to save cost and time for the users, at the same time, it also can reduce the pressure on urban traffic, maximize the use of road resources, so the research of 
dynamic navigation path selection problem really has important practical values and theoretical significances.

Intelligent navigation dynamic path planning is based on the topology relationship between the city's road networks, historical traffic information and real-time traffic information to plan the optimal travel routes in accordance with the requirements of travelers, thus providing the most satisfying travel policy for travelers in order to improve the utilization of road resources, reduce vehicle trips in the waiting time, reduce vehicle exhaust emissions, protect the environment, and ultimately to ease the traffic pressure on the city to provide security for the safe operation of the urban road system. Major significances of intelligent navigation dynamic path are as follows:

A. Customize personalized optimal route. According to the weight of time, distance, and cost given by travelers, optimal travel route in accordance with the requirements of traveler is provided. Such as minimum time, shortest distance, minimum cost, or time, distance, cost of each account for certain proportion.

B. Relieve traffic congestion and safeguard the traffic safety. Dynamic path planning for users can avoid traffic congestion, traffic accidents, and temporary traffic controlling order to make the traffic flow tends smooth, protect the safety of travelers, reduce the incidence of traffic accidents effectively, and improve the safety 1 of the whole urban traffic network.

C. Improve transport efficiency and increase economic benefits. For enterprises, logistics of high speed can improve the efficiency, thus contributing to improve economic efficiency. For individuals, a convenient, smooth, fast trip which save their time and bring them a good attitude, which can be reflected in their respective work, and the resulting economic benefits is immeasurable.

\section{Current Research Status of the Path Planning Problems}

At present, vehicle navigation systems used in the market are basically about static path planning and cannot change the route according to real-time traffic accidents, traffic jams and so on [4-6]. However, faced with the reality of unstable traffic, the static route is unable to meet the needs of users. Therefore, the dynamic path planning has been becoming a hot topic in new generation of intelligent vehicle navigation systems. As the road grade, distance, time, cost and some other information are stored as section properties in digital road maps, and then first of all, the digital map should be converted into a weighted directed graph [7-9]. Therefore, the problem of calculate the optimal path between any two points in the road network can boil down to solve the optimal problem of the weighted directed graph. There are many more mature shortest path algorithms to choose from, but in the vehicle navigation system, limited by the car environment and cost, processing power and storage resource, these algorithms are usually cannot be used directly in graph theory. At the same time, the real-time dynamic path planning has higher demand of the real-time characteristic, and that means it requires higher operational efficiency. To this end, by improving the operation 
efficiency of existing algorithm or constructing new efficient algorithm to meet the timeliness requirements of vehicle navigation can tackle these problems.

\section{The Basic and Improved Ant Colony Algorithm}

\subsection{Basic Ant Colony Algorithm}

Ant colony algorithm is a new kind of random search simulated evolutionary algorithm, put forward by the Italian scholar M. Dorig, V. Maniezzo in 1991, who imitate of ant colony behavior in the real world [6]. Ant colony algorithm is first successfully applied to the Travelling Salesman Problem (TSP) problem, a simple description of the traveling salesman problem is: among the given cities, there is a travelling salesman starting from a certain city, after each city once, and only once, return to the original departure city again, a shortest path tour is needed. Here we take the TSP problem as an example, a brief introduction of the basic algorithm [5-7].

Build a city set on graph theory, described as follows: Given a graph $G=(V, A)$, where $V$ represents the figure points set, means various cities. A indicates that the connection points of the edge set, means inter-city roads. Then a shortest path through the entire city is required. Well, the objective function of the problem is (1):

$$
\min D=\sum_{i=1}^{n} d(i, i+1)+d(n, 1)
$$

where $n$ represents $n$ cities; denotes from the $i^{\text {th }}$ city to an the $(i+1)^{\text {th }}$; means the distance between the first city and the last city . To find the optimal solution of TSP problem, the following is the established model of ant colony algorithm.

Suppose there are $m$ ants, $\tau_{i j}(t)$ represents time with the information concentration of the side $(i, j)$ on the time $t$; indicates the number of ants in the city $i$ on the time $t \cdot d_{i j}(i, j=$ $1,2, \cdots n$ ) represents the distance from city $i$ to $j ; \eta_{i j}$ indicates intensity of heuristic information of edge $(i, j)$. In the basic ant colony algorithm:

$$
\mathrm{m}=\sum_{\mathrm{i}=1}^{\mathrm{n}} \mathrm{b}_{\mathrm{i}}(\mathrm{t})(\mathrm{i}=1,2, \cdots \mathrm{n})
$$

$$
\eta_{i j}=1 / d_{i j}
$$

$\alpha$ is inspired factor for the information, it means the amount of information the ants during the reaction process in motion relative. $\beta$ indicates expect heuristic factor, reflecting the relative importance of heuristic information in guiding the search process of ant.

Assumed that $\mathrm{m}$ ants be placed into $\mathrm{n}$ randomly selected cities, each ant find the next city according to the rules; As soon as the city is found, the information for residual concentration 
$\tau_{\mathrm{ij} \text { ( } t)}$ is updated. According to the principle above, the ant keeps on selecting the next city:

Initially, there are no pheromones on the road, set $\tau_{\mathrm{ij}}(0)=\mathrm{C}$ (C is constant).the ant start from a city, and choose the next city according to the state transition rules, the RLE is also called "random ratio rule". $\mathrm{p}_{\mathrm{ij}}^{\mathrm{k}}(\mathrm{t})$ is the probability of ant $\mathrm{k}$ transferred from $\mathrm{i}$ city to city $\mathrm{j}$ at time t, just as formula (4) says.

$$
\mathrm{p}_{\mathrm{ij}}(\mathrm{t})=\left\{\begin{array}{cc}
\frac{\tau_{\mathrm{ij}}^{\alpha}(\mathrm{t}) \eta_{\mathrm{ij}}^{\beta}(\mathrm{t})}{\sum_{\mathrm{keallowed}} \tau_{\mathrm{ik}}^{\alpha}(\mathrm{t}) \eta_{\mathrm{ik}}^{\beta}(\mathrm{t})}, \mathrm{j \in \text {allowed } _ { \mathrm { k } }} \\
0, \text { else }
\end{array}\right.
$$

allowed $_{\mathrm{k}}=[1,2, \cdots \mathrm{n}]-$ tabu $_{\mathrm{k}}$ represents the city tables of allowed ant $\mathrm{k}$ to select in the next step. tabu $\mathrm{k}_{\mathrm{k}}$ records the city which ant $\mathrm{k}$ has traversed, updating with the evolution of ant $\mathrm{k}$. Cities in the table are not allowed to select, $\mathrm{K}$ represents the cities which ant $\mathrm{k}$ can choose, that is to say, the city is not in the table of tabu $\mathrm{k}_{\mathrm{k}}$. Ants move forward in accordance with the rules. The iteration is complete until all ants find the path. Then the pheromone can be used to update the pheromone on the path, and then start the next iteration until the maximum number of iterations. Pheromone update rule is:

$$
\tau_{i j}(i+1)=(1-\rho) \tau_{i j}(t)+\Delta \tau_{i j}
$$

$$
\begin{aligned}
& \Delta \tau_{\mathrm{ij}}=\sum_{\mathrm{k}=1}^{\mathrm{n}} \Delta \tau_{\mathrm{ij}}^{\mathrm{k}} \\
& \Delta \tau_{\mathrm{ij}}^{\mathrm{k}}=\left\{\begin{array}{c}
\frac{\mathrm{Q}}{\mathrm{L}_{\mathrm{k}}}, \text { if the } \mathrm{k}^{\mathrm{th}} \text { ant through } \mathrm{i} \mathrm{j} \text { in this cysle } \\
0,
\end{array}\right.
\end{aligned}
$$

Where $0<\rho<1$, showing persistence track; $1-\rho$ said reduction of track, that the degree of disappearance of information. $\mathrm{Q}$ is a constant, $\mathrm{L}_{\mathrm{k}}$ represents length of travel path length of the week. This $\Delta \tau_{i j}$ represents an increase of the amount of information during the iteration in path $(\mathrm{i}, \mathrm{j})$. According to $\mathrm{L}_{\mathrm{k}}$, the shorter the path is, the more pheromone it releases; $\Delta \tau_{\mathrm{ij}}^{\mathrm{k}}(\mathrm{t})$ represents the amount of information what ant $\mathrm{k}$ remain in the path $(\mathrm{i}, \mathrm{j})$ in the iteration.

\subsection{Improved Ant Colony Algorithm}

Regard the user's multi-demands as a condition of pheromone update [5]: 


$$
\Delta \tau_{\mathrm{ij}}(\mathrm{t})=\sum_{\mathrm{k}=1}^{\mathrm{n}} \Delta \tau_{\mathrm{ij}}^{\mathrm{k}}(\mathrm{t})=\left\{\begin{array}{c}
\sum_{\mathrm{k}=1}^{\mathrm{n}} \frac{\mathrm{Q}}{\mathrm{f}}, \text { if the } \mathrm{k}^{\text {th }} \text { ant through } \mathrm{i} \mathrm{j} \text { in this cycle } \\
0,
\end{array}\right.
$$

(8) Among this:

$$
f=w_{1} f_{1}+w_{2} f_{2}+w_{3} f_{3}+w_{4} f_{4}+w_{5} f_{5}
$$

(9) $f_{1}, f_{2}, f_{3}, f_{4}, f_{5}$ respectively represents the entire cost, distance, travel time, road capacity and road grade information of the section of $\mathrm{C}_{\mathrm{ij}} \cdot \mathrm{w}_{1}, \mathrm{w}_{2}, \mathrm{w}_{3} \ldots$ respectively corresponding to the weight in heuristic function. Meanwhile, the intensity of heuristic information function not only considers the distance between $d_{i j}$ and $C_{i j}$, but also considers distance of the follow-up $d_{i j}$ in order to avoid the algorithm trapping into local optimal solution, So the improved intensity of heuristic information function is (10):

$$
\eta_{\mathrm{ij}}=\frac{1}{\min [\mathrm{d}(\mathrm{i}, \mathrm{j})+\mathrm{d}(\mathrm{j}, \mathrm{n})]}
$$

(10) Then

$$
\mathrm{p}_{\mathrm{ij}}^{\mathrm{k}}=\left\{\begin{array}{cl}
\frac{\tau_{\mathrm{ij}}^{\alpha} \eta_{\mathrm{ij}}^{\beta}}{\sum_{\mathrm{k} \in \text { allowed }_{\mathrm{k}}}^{\beta} \tau_{\mathrm{ik}}^{\alpha} \eta_{\mathrm{ik}}^{\beta}} & , \text { if } \mathrm{j} \in \text { allowed }_{\mathrm{k}} \\
0 & , \text { else }
\end{array}\right.
$$

(11) When the pheromone intensity was less than a certain value, the ant colony algorithm convergence speed would not be fast. Otherwise, the convergence speed could be really quickly, unfortunately, the algorithm's global search ability would become worse, and would fall into local optimal solution much easier. Therefore, we should set a certain threshold $\mathrm{q}_{0}$, when did not reach it, the ant will ignore the impact of the stimulus automatically until the threshold is reached.

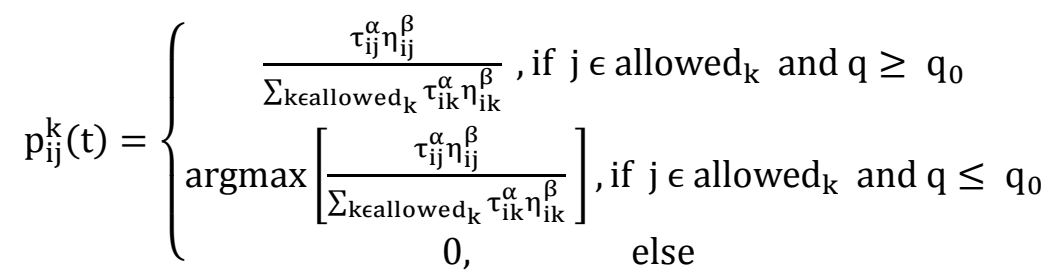

Ant colony algorithm flowchart is shown in Figure 1. 


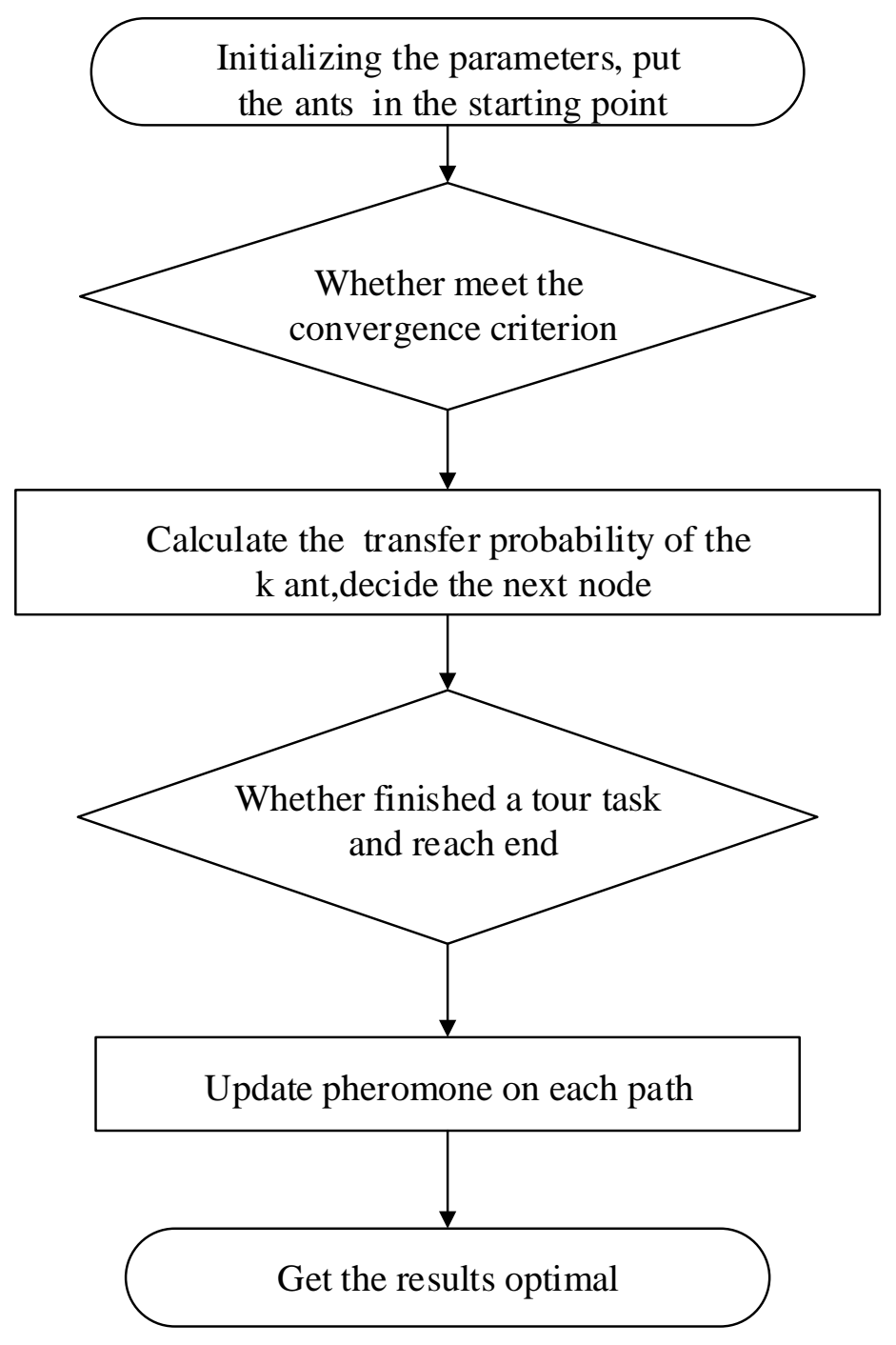

Figure 1. Path Ant Colony Algorithm

\subsection{The Optimal Routing Selection based on Ant Colony Algorithm}

The issues of optimal path choice of transportation are similar to ants foraging behavior. Regard the starting point of the vehicle as an ant nest, destination as the source which the ants are looking for .Vehicles from the starting point, passed some sections, reached the final destination. And during the time, vehicles choose the routine according to their own judgments. The entire road network is seen as a directed graph, the vehicle is the artificial ants with intelligent behavior, what is more, the artificial ants just find a path from the starting point to the end; need not return to the starting point. By abstracting the process of transportation, regard the actual cost of transportation as heuristic information can build an artificial ant colony system of optimal path selection. Figure 2 shows the algorithm steps of ant colony algorithm in the select of actual path. 


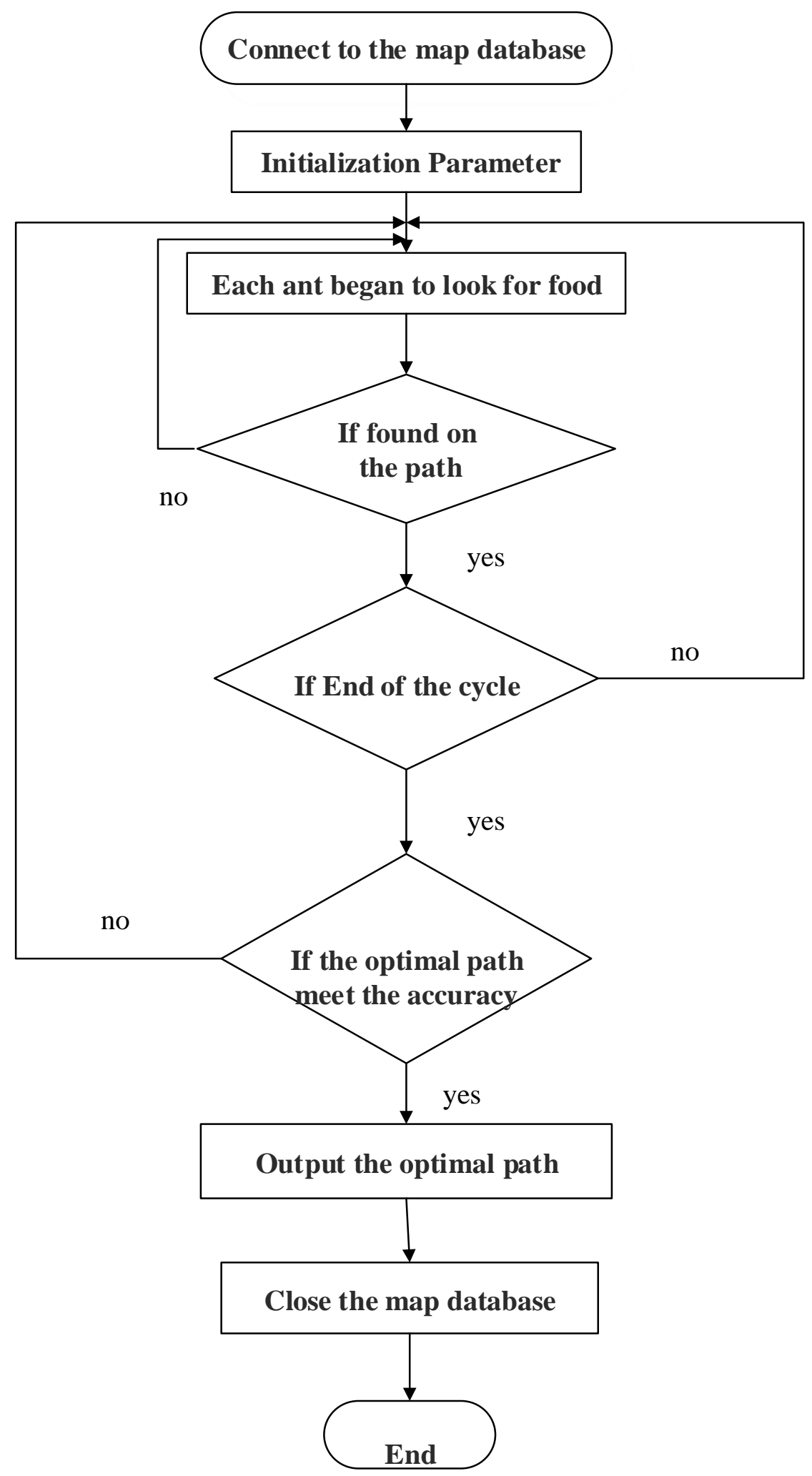

Figure 2. The Flow Chart Path Selection based on Ant Colony Algorithm 


\section{Case Study}

\subsection{Targets}

Currently, the using vehicle navigation systems in the market are basically static path planning and cannot change the route according to traffic information (traffic flow, reconstruction) and abnormal events (traffic accident, important event) and so on [17-18]. And it can only provide a path to meet the single "optimal" conditions, such as the minimum time path, the shortest distance path, failing to coordinate users' multi-demand for path. Systems such as Baidu, AutoNavi and so on provides information on traffic conditions and traffic forecasts, but failing to use well in path planning. The provided navigation routes of the least time or shortest distance just considers whether taking the Highway or not, but not based on traffic congestion, traffic control, travel expenses, etc. [17-20]. Some other navigation systems may be considered these, but these are getting before travelers set off, do not represent the traffic situation when travelers arrive. Traffic jams at the beginning don't mean that there is a traffic jam when they arrive. To address the above problem, our research is that traveler set the optimal path constraint and the corresponding weights firstly. For example, the proportion of the cost of travel is $30 \%, 40 \%$ of travel distance, travel time by $20 \%$. Then by mining historical data, predict time of arrival in a road, predict the traffic condition when arrived, and then according to the predicted traffic to plan route. At the same time we will judge whether the current traffic situation is consistent with the forecast traffic conditions according to real-time traffic information every 5 minutes, and make the appropriate adjustments. When the route has changed, remind the user to change the route [18-20]. Figure 3 and Figure 4 respectively is Baidu map provides the minimum time path and the shortest distance path from Wulin Square to Hangzhou East Railway Station.

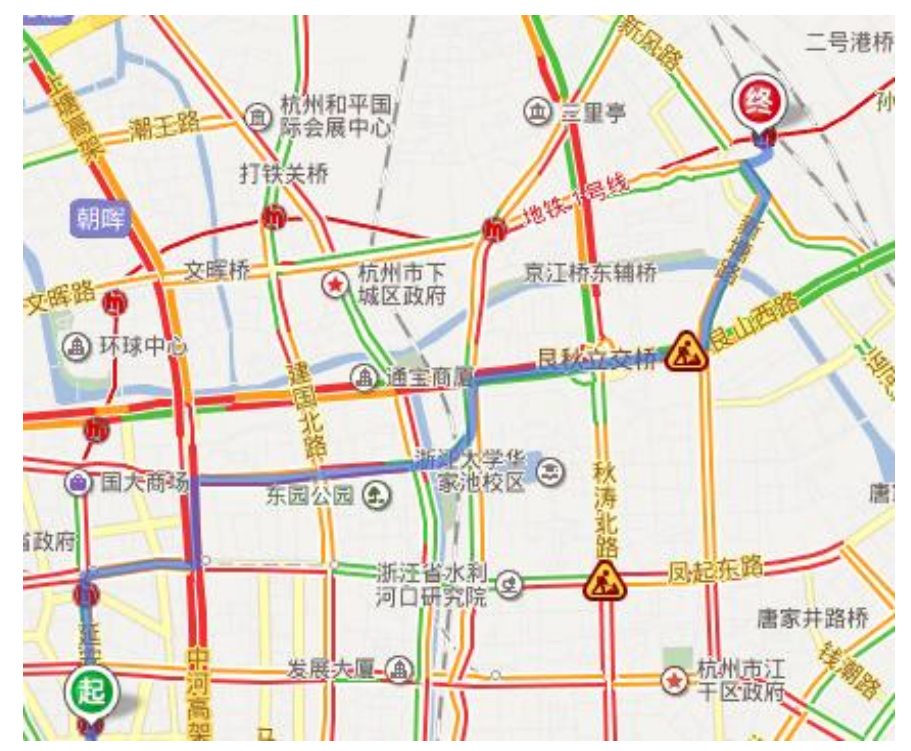


Figure 3. The Minimum Time Path from Wulin Square to Hangzhou East Railway Station

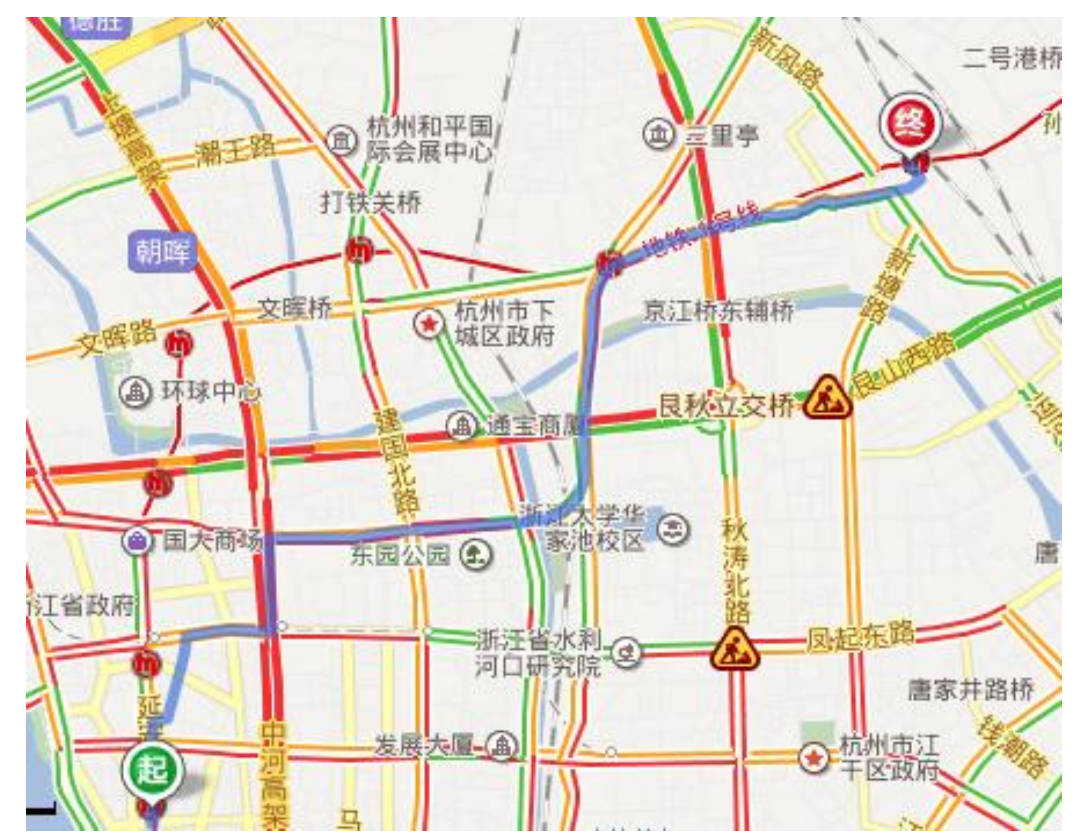

Figure 4. The Shortest Distance Path from Wulin Square to Hangzhou East Railway Station

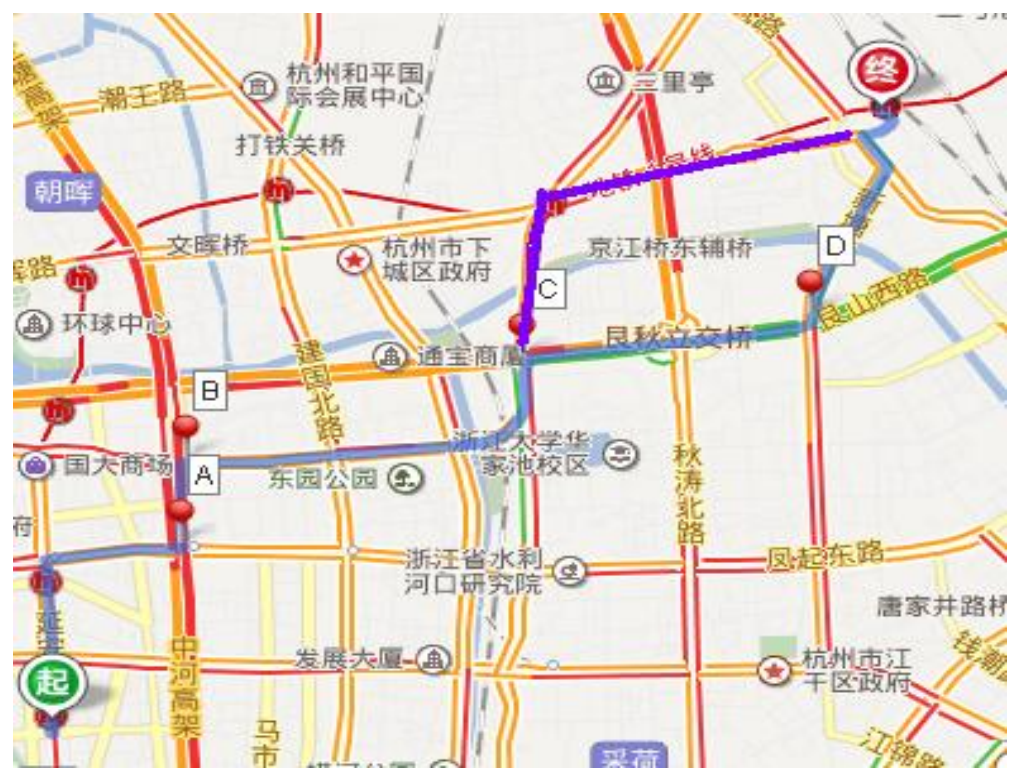

Figure 5. The Optical Path Planning

Just as Figure 5 shows, travelers query the start and end of the path when they set off. The path we are planning is not according to the traffic flow at this time, but traffic conditions we 
forecast, such as A to B, though when traffic conditions are severe blockage at that time, if we predict that traffic is flowing when the traveler arrives at point $\mathrm{A}$, the path planning would not avoid this road. When the vehicle leaves point at about $100 \mathrm{~m}$, prejudgments of the road ahead whether it is consistent with our forecast based on real-time traffic conditions. When the vehicle reaches the point $\mathrm{C}$, the originally unobstructed road may be blocked, so we have to make an adjustment again, which may be different from the planned one as purple path shown.

\subsection{Simulation and Analysis}

In order to verify the effectiveness and feasibility of the algorithm, the algorithm is applied to a simple network simulation model, determining the effectiveness of the algorithm through the simulation results.

Firstly, establish the map database used in the simulation experiment in MapInfo, in this simulation experiment, nodes represent road junctions in the city, edges represent roads between nodes. And individual ID of each node along with the corresponding coordinates will be stored as node data information, and the node table is shown in Table 3-4.Road simulation information includes the road of start-stop node information, road length, road grade and road speed, the road sides table is shown in Table 1 and Table 2.

Table 1. Node Table

\begin{tabular}{|c|c|c|}
\hline ID & $X$ & $Y$ \\
\hline 1 & 7 & 18 \\
\hline 2 & 8 & 10 \\
\hline 3 & 12 & 14 \\
\hline 4 & 13 & 27 \\
\hline 5 & 17 & 22 \\
\hline 6 & 23 & 20 \\
\hline
\end{tabular}

Table 2. Road Sides Table

\begin{tabular}{|c|c|c|c|c|c|}
\hline ID & SPoint & EPoint & Length & Grade & Speed \\
\hline 1 & 1 & 2 & 30.8 & 1 & 30 \\
\hline 2 & 1 & 3 & 29 & 2 & 35 \\
\hline 3 & 1 & 4 & 55.6 & 1 & 28 \\
\hline 4 & 3 & 6 & 40.4 & 2 & 40 \\
\hline 5 & 3 & 5 & 47.6 & 2 & 18 \\
\hline 6 & 6 & 5 & 36.5 & 230 & 36 \\
\hline
\end{tabular}

The simulation results calculate a simple path at the minimum time, the shortest distance and multi-constrained dynamic programming. The results are shown in Figure 6. The shortest distance path is 1, 3, 6, 9 and 12. The Minimum time path is 1, 2, 3, 6, 9 and 12. After considering the road traffic congestion by using ant colony algorithm, the weight of time, distance, road grade and full payment respectively are $20 \%, 40 \%, 20 \%$ and $20 \%$, so the multi-constrained dynamic path will be $1,3,6,5,8$ and 12 . 


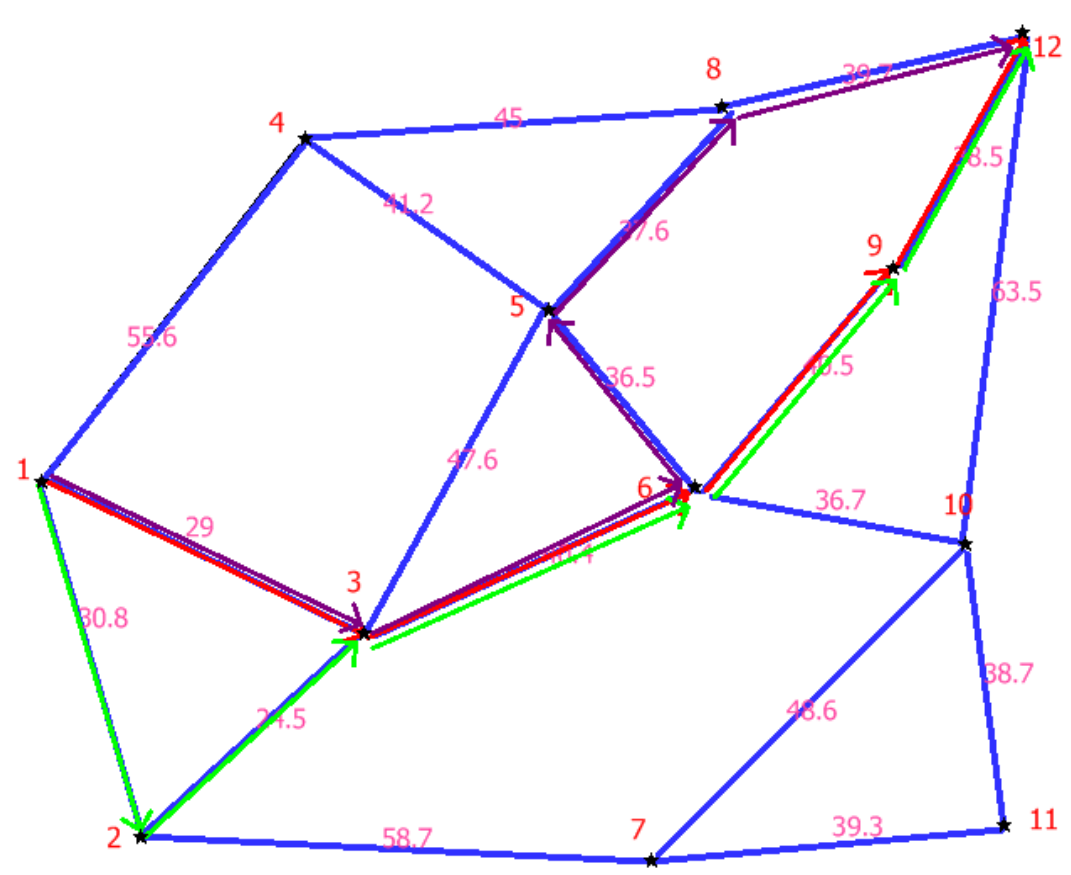

Figure 6. Path Planning and Optimization

\section{Conclusions}

Nowadays, the urban traffic is becoming more and more complicated, increasing vehicles 、 road maintenance、 traffic congestion and other issues have brought a great burden to the current traffic system, just the control of urban transportation center is no longer enough to solve the increasingly serious traffic problems. The vehicle navigation system can effectively alleviate the urban traffic congestion, optimization of urban traffic flow, improve transport efficiency, and reduce environmental pollutions and so on. Dynamic path planning is the core technology in vehicle navigation system, which is also the precondition of realizing other navigation functions accurately. And the path algorithm is the key factor of the optimal path.

The strategy is the traveler can establish the path selection for multi constrained programming model according to their actual needs, giving time, cost, and distance their corresponding proportion. And then through the cleaning treatment on historical traffic data, and then train the processed data by time interval, according to the requirements of the traveler optimal travel route, the training results predict the same time traffic flow. The advantage of this algorithm is in the running process, the future traffic forecast information on travel time and distance considering route guidance scheme, at the same time, according to the traffic information, it also periodically dynamic update in real-time for travelers to provide the optimal route to the new, but also the voice prompt travelers whether to change the route or not, finally design the most consistent with the actual traffic demand traffic guidance scheme. 


\section{Acknowledgements}

The authors wish to thank the National Natural Science Foundation of China for contract 61100194, the Public Projects of Zhejiang Province for contract 2013C33082, the foundation of education department of Zhejiang province of China for contract Y201120520 and the research foundation of Hangzhou Dianzi University for contract KYS105612008 and YB1205, under which the present work was possible.

\section{References}

[1] D. Seng, H. Wu, X. Fang and H. Xu, "Information fusion of ITS based on granular computing”, Journal of Chemical and Pharmaceutical Research, vol. 5, no. 12, (2013), pp. 553-559.

[2] H. Wu, D. Seng, X. Fang and H. Xu, "Application of information fusion technologies for multi-source data", Journal of Chemical and Pharmaceutical Research, vol. 5, no. 12, (2013), pp. 560-564.

[3] X. Fang, H. Wu, D. Seng and H. Xu, "Traffic flow data mining based on cloud computing", Journal of Chemical and Pharmaceutical Research, vol. 5, no. 12, (2013), pp. 565-569.

[4] S. Moafipoor, L. Bock, J. A. Fayman, G. Mader and P. J. De Jonge, :Development and assessment of a low dynamic vehicle navigation system”, Institute of Navigation - International Technical Meeting 2011, ITM 2011, vol. 2, (2011), pp. 895-907.

[5] A.-M. Khalid, A.-S. Mansour, E. Muhammad and R. Hedjar, "D lite based real-time multi-Agent path planning in dynamic environments", Proceedings of the ISCA 24th International Conference on Computer Applications in Industry and Engineering, CAINE 2011, (2011), pp. 124-128.

[6] M. Dorigo and L. M. Gambardella, "Ant colony system: a cooperative learning approach to the traveling salesman problem”, IEEE Transactions on Evolutionary Computation, vol. 1, (1997) April, pp. 53-66.

[7] B. Bullnheimer, R. F. Hartl and C. Strauss, "An improved ant system algorithm for the vehicle routing problem", Annals of Operations Research, vol. 89, (1999), pp. 319-328.

[8] H. Beckmann, B. Eissfeller and Robust, "Secure and precise vehicle navigation system for harsh GNSS signal conditions", 25th International Technical Meeting of the Satellite Division of the Institute of Navigation 2012, ION GNSS 2012, vol. 3, (2012), pp. 1589-1600.

[9] B. X. Weis and A. Sandweg, "GuideWeb: A conceptually infrastructure-free vehicle navigation system", IET Intelligent Transport Systems, vol. 6, no. 2, (2012) June, pp. 139-144.

[10] D. Seng, "Application of computer in material science and engineering", Applied Mechanics and Materials, vol. 189, (2012), pp. 482-485.

[11] D. Seng and Z. Li, "Design and implementation of a 3D simulation system for geological and mining engineering”, Journal of Liaoning Technical University, vol. 27, no. 1, (2008) February, pp. 9-12.

[12] D. Seng and Y. Shu, "Framework and construction contents of digital mine", Advances in Intelligent Systems and Computing, (2013), pp. 393-400.

[13] D. Seng, "Visualization of composite materials' microstructure with OpenGL", Applied Mechanics and Materials, vol. 189, (2012), pp. 478-481.

[14] D. Seng, "Simulation techniques in the research of structure and performance of nanosized materials", Applied Mechanics and Materials, vol. 189, (2012), pp. 457-460. 
[15] D. Seng and Y. Shu, "Research on the application and status quo of digital mine and sensing mine", Advances in Intelligent Systems and Computing, (2013), pp. 401-408.

[16] W. Chen, S. Zhu and D. Li, "Vehicle-assisted shortest-time path navigation", 2010 IEEE 7th International Conference on Mobile Adhoc and Sensor Systems, MASS 2010, (2010), pp. 442-451.

[17] R. A. Abbas and H. M. Mahdi, "Using finite elements method for traffic flow analysis", ICTIS 2011: Multimodal Approach to Sustained Transportation System Development - Information, Technology, Implementation - Proceedings of the 1st Int. Conf. on Transportation Information and Safety, (2011), pp. 1131-1136.

[18] G.-R. Edgar, A. Y. Alanis, N. Arana-Daniel and C. Lopez-Franco, "Integration of an inverse optimal control system with reinforced-SLAM for path planning and mapping in dynamic environments", 2013 IEEE International Autumn Meeting on Power, Electronics and Computing, ROPEC 2013, (2013).

[19] J.-Y. Bai and S.-Y. Li, "Improved ant colony algorithm with emphasis on data processing and dynamic city choice”, Proceedings - 2009 International Conference on Information Engineering and Computer Science, ICIECS 2009, (2009).

[20] A. Sud, E. Andersen, S. Curtis, M. C. Lin and M. Dinesh, "Real-time path planning in dynamic virtual environments using multiagent navigation graphs", IEEE Transactions on Visualization and Computer Graphics, vol. 14, no. 3, (2008) May-June, pp. 526-538. 
International Journal of $u-$ and e-Service, Science and Technology Vol. 7, No. 6 (2014) 